\title{
A Practical Approach to Introducing Pre-hospital Resuscitative Endovascular Balloon Occlusion of the Aorta (REBOA), the Problems Encountered and Lessons Learned
}

\author{
Manik Chana' ${ }^{1}$ Zane Perkins ${ }^{2}$, Robbie Lendrum ${ }^{3}$ and Samy Sadek ${ }^{1}$ \\ 'The Institute of Pre-hospital Care at London's Air Ambulance, London, UK \\ ${ }^{2}$ The Royal London Hospital Major Trauma Centre, Barts Centre for Trauma Sciences, London, UK \\ ${ }^{3}$ Royal Infirmary of Edinburgh, UK
}

\begin{abstract}
Resuscitative Endovascular Balloon Occlusion of the Aorta (REBOA) is an endovascular procedure which utilises a catheterbased balloon device to achieve aortic occlusion. The aim of this resuscitative measure is to improve blood pressure proximal to the occlusion site and therefore preserve cardiac and cerebral perfusion to prevent cardiac arrest. In addition, there is a relative reduction in arterial flow to the site of injury distal to the balloon. Endovascular techniques are gaining acceptance for the in-hospital management of haemorrhage; however, their use in pre-hospital care is still limited. This is due to several factors including the technical challenges, training and skill sets of pre-hospital care teams and the potential for harm of REBOA, particularly with extended balloon occlusion times. However, non-compressible torso haemorrhage is associated with a mortality of approximately $50 \%$ and a significant proportion of these deaths occur in the pre-hospital phase of care. In the exsanguinating patient, resuscitative thoracotomy with direct aortic compression is often the only means to control haemorrhage. This resuscitative measure is now a more established pre-hospital intervention which has significantly improved outcomes in the context of penetrating trauma, particularly thoracic injury. However, in the context of blunt injury and subdiaphragmatic haemorrhage, the outcomes from pre-hospital resuscitative thoracotomy remain poor. We present our initial technique for successfully introducing REBOA for the pre-hospital management of exsanguinating pelvic or groin haemorrhage following trauma, our indications for REBOA and comments on the problems and limitations encountered, as well as the lessons learned.
\end{abstract}

Keywords: REBOA; Resuscitation; Trauma; Haemorrhage; Pre-hospital Care; Endovascular

\section{INTRODUCTION}

Non-compressible torso haemorrhage (NCTH) is the leading cause of potentially preventable trauma deaths [1]. Junctional vascular injuries and pelvic fractures resulting from blunt and penetrating mechanisms of

\section{Corresponding author:}

Manik Chana, The Institute of Pre-hospital Care at London's Air Ambulance, The Helipad, The Royal London Hospital, Whitechapel, London E1 1BB, UK.

Email: manik.chana@nhs.net

(C) 2021 CC BY-NC 4.0 - in cooperation with Depts. of Cardiothoracic/Vascular Surgery, General Surgery and Anesthesia, Örebro University Hospital and Örebro University, Sweden injury form a major part of NCTH. These injuries can lead to exsanguinating haemorrhage resulting in traumatic cardiac arrest with reported mortality rates of up to $50 \%$ [2-4]. A peak in mortality is observed in these patients at around 30 minutes from injury which, for a large proportion of patients, occurs in the pre-hospital environment [5]. London's Air Ambulance (LAA) is a physician-led pre-hospital service providing an advanced trauma team which aims to deliver effective treatment to critically injured patients as soon as possible following injury. Recent data show that mortality in shocked patients undergoing laparotomy is unchanged despite advances in trauma care [6]. Resuscitative thoracotomy and aortic compression (RT) is an open surgical technique which is well established in the hospital, and its 
use has been described in the treatment of traumatic cardiac arrest by non-surgeons in the pre-hospital phase of care $[7,8]$. It is effective for penetrating injuries with cardiac tamponade or a thoracic source of haemorrhage [9]. However, RT carries major surgical morbidity and has poor outcomes in blunt injury and subdiaphragmatic haemorrhage $[10,11]$.

We recognised that for a meaningful reduction in mortality in patients with subdiaphragmatic exsanguination, novel resuscitation solutions were required. In 2013, LAA delivered the first known civilian prehospital resuscitative endovascular balloon occlusion of the aorta (REBOA) [4], and since then we have continued to successfully develop this intervention [12]. This was developed using 'off the shelf' and readily available equipment used for routine endovascular procedures. The aims of this article are to describe the practical technique which was initially adopted to successfully perform pre-hospital REBOA as a resuscitation adjunct following major trauma, to describe our indications for REBOA and to comment on the problems and limitations encountered. We also consider how this equipment and technique could be implemented as an endovascular resuscitation and haemorrhage control service in austere healthcare systems and its potential to be used in the management of postpartum haemorrhage [13] and out-of-hospital cardiac arrest [14].

\section{REBOA IN PRE-HOSPITAL CARE}

The use of endovascular techniques is becoming more prevalent in the in-hospital management of trauma haemorrhage [4,15-20]. REBOA involves inflation of a compliant balloon within the aorta to provide temporary occlusion and control of bleeding inflow to the site of injury prior to definitive haemostasis. This limits ongoing haemorrhage and preferentially supports myocardial and cerebral perfusion, albeit at the expense of distal ischaemia. These principles are in keeping with RT and aortic cross clamping but achieved in a less invasive manner in the already injured patient [20-25]. In traditional endovascular practice, vascular access is gained via the common femoral artery (CFA), ideally under ultrasound scan (USS) guidance or via direct surgical exposure, and devices are positioned and deployed under fluoroscopy in the controlled and sterile environment of an operating theatre, interventional radiology suite or hybrid theatre. However, the rapid onset of exsanguinating haemorrhage and expected mortality associated with NCTH has required REBOA to be performed in more austere conditions encountered in pre-hospital care and by physicians from backgrounds other than vascular surgery or interventional radiology. The aims of pre-hospital REBOA are to permit resuscitation, rapidly control exsanguinating haemorrhage and to transport to definitive care.
The aorta can be divided into three distinct zones [26]. Zone I extends from the origin of the left subclavian artery to the coeliac axis. Zone II extends from the coeliac axis to the most caudal renal artery. Zone III is distal from the most caudal renal artery to the aortic bifurcation. A multidisciplinary group with expertise from emergency medicine and pre-hospital care, trauma surgery, interventional radiology, anaesthetics and intensive care medicine began developing a standard operating procedure (SOP) for pre-hospital zone III REBOA in 2012. It was agreed that basic measures should be performed first including non-invasive haemorrhage control techniques such as minimal handling, pelvic splinting, limb splinting, tourniquets, transfusion of blood products and antifibrinolytics, and rapid transfer to a Major Trauma Centre (MTC). At the time of development, it was anticipated that balloon occlusion times would be in excess of 30 minutes from inflation to definitive haemorrhage control. For this reason, REBOA would be limited only to zone III in order to minimise the risk of visceral ischaemia. In addition to this, we felt that femoral puncture site morbidity should be limited by using an $8 \mathrm{Fr}$ sheath as a maximum. At this time, there was a lack of bespoke lower profile endovascular devices with a balloon large enough to occlude the aorta in zone I which were compatible with this sheath size, precluding more proximal balloon occlusion. These were the key factors in establishing the equipment required to safely implement a strategy for zone III REBOA in the pre-hospital environment.

At present there is no universally agreed definite indication for pre-hospital REBOA and the population most likely to benefit from the procedure has yet to be clearly defined. The in-hospital use of REBOA is currently the subject of an ongoing study [27]. We feel the use of pre-hospital zone III REBOA is indicated in the context of blunt or penetrating injury to the pelvis or groin, when a senior, experienced clinical team identify unequivocal signs of true exsanguinating haemorrhage and imminent risk of hypovolaemic cardiac arrest [28].

\section{Ethical Approval and Informed Consent}

Ethical approval was not required. No identifiable data is included.

\section{LAA TECHNIQUE FOR PRE-HOSPITAL REBOA}

The technical performance of REBOA can be challenging and becomes even more so when performed in time critical situations out-of-hospital in an austere environment. The layout of the REBOA pack carried by LAA initially focussed primarily on size and weight but also incorporated features to aid the clinician and assistant performing the procedure. The packs contained sections numbered from one to four, in the order that they were 
to be used, and the equipment was chosen to be as minimal and intuitive as possible. With subsequent development this was improved with the introduction of bespoke REBOA packs. The equipment and technique described here was utilised for all patients in our initial case series [8].

\section{Patient Selection and Positioning}

Patient selection is based on decision making by senior experienced doctors as part of the LAA team according to previously established criteria suggesting exsanguinating pelvic haemorrhage [28] (Table 1). When establishing this service, no age limits were specified but our SOP was only to consider pre-hospital REBOA in adult patients. REBOA is only considered in cases where the impression of these senior clinicians is that the trajectory of presenting physiology is imminent cardiovascular collapse and traumatic cardiac arrest, with an injury pattern congruent with this physiology.

The patient should be positioned supine in an area with 360-degree access and all efforts made to calm, protect and optimise the immediate area around the clinician and assistant performing the procedure. Concomitant tasks, such as intravenous access and intubation/ ventilation for example, should be performed by other members of the team and a separate team member should take over the role of team leadership while the intervening clinicians are task focussed. In patients with suspected pelvic fractures, clothing should be removed and a pelvic splint should be applied. It may be necessary to carefully move or modify the binder to allow femoral vascular access. Prepare the skin and place the drape over the patient, exposing the operative area of interest.

\section{Arterial Access}

Arterial access should be gained via the CFA. In patients with a suspected junctional vascular injury, the contralateral CFA should be used. The position of the CFA can be estimated at a point approximately $2-\mathrm{cm}$ inferior to the mid-inguinal point.

The femoral vascular structures should be visualised under USS with pulsatile flow seen to confirm the CFA. Puncture the CFA under USS guidance using a percutaneous entry thin-walled needle (Cook, Indianapolis, USA) or in severely shocked patients using a Merit MAK mini access kit (Merit Medical, Utah, USA). Access is then established by passing an 0.035 -inch guidewire into the needle followed by a 7Fr sheath (Cordis, California, USA) with its dilator on the wire. With a skin incision made this should advance into the artery without resistance. Ensure the sheath's side port is in the 'off' position. On completion of this step, there will be an $11-\mathrm{cm}$ 7Fr sheath providing access to the CFA and external iliac artery providing a port through which REBOA can be performed.

\section{Balloon Positioning and Inflation}

Pass the Amplatz Extra-Stiff guidewire (Cook, Indianapolis, USA) with j-tip through the sheath to a depth of approximately $50 \mathrm{~cm}$ from the skin incision. The guidewire should pass without resistance if in the correct intraluminal position. Perform a test inflation of the balloon with the pre-loaded $2.5 \mathrm{-ml}$ syringe of saline provided. Load the balloon catheter (LeMaitre, Massachusetts, USA) onto the guidewire and advance to a depth of $40 \mathrm{~cm}$ from the skin. Take care to ensure the wire is not withdrawn and remains sterile. Inflate the balloon with $0.9 \% \mathrm{NaCl}$ to a maximum of $2.5 \mathrm{ml}$ or until resistance is encountered. This balloon will enlarge approximately to a $14-\mathrm{mm}$ diameter when inflated with $2.5 \mathrm{ml}$. Clearly document the time of balloon inflation.

Once the balloon is inflated, aortic pulsation should push it towards the terminal aorta (if aortic pressure is high enough) and it will be supported at the bifurcation. Allow the balloon to migrate spontaneously or apply very gentle caudal traction if required. Confirm correct positioning with an improvement in central haemodynamic, absence of lower limb pulses and absence (or marked reduction) of pulsatile flow on USS distal to the balloon.

The sheath should then be sutured in position and the catheter, sheath and wire covered with an adhesive dressing. A team member should be tasked with maintaining the position of the device and wire.

After REBOA, all efforts should be concentrated on minimising balloon occlusion time. Practically speaking, this involves immediate transfer for definitive care at an appropriate MTC during which there is a focus on volume resuscitation.

Following balloon inflation, patients may become normotensive or even hypertensive as a result of the increased systemic vascular resistance and improved coronary perfusion. A return of cerebral perfusion may necessitate review of adequate analgesia or depth of anaesthesia. As resuscitation continues, increasing aortic pressures may now cause a balloon which had not migrated back to the bifurcation to do so. Therefore, careful attention must be paid at all times to ensure the endovascular device is secured.

On arrival in the Emergency Department, clear handover should be made to the receiving trauma team including the presence of REBOA, balloon inflation time and the need for prompt haemorrhage control emphasised. All processes should function to minimise the total time of balloon inflation and distal ischaemia.

\section{Balloon Deflation and Sheath Removal}

Balloon deflation and catheter removal should occur in a controlled environment in a planned coordinated manner under instruction of a trauma team leader and with input from anaesthetics and interventional radiology or 
trauma/vascular surgery. It is important to be aware that profound haemodynamic instability may follow balloon deflation due to a sudden reduction in systemic vascular resistance, hypovolaemia, reperfusion and washout of metabolic products with hyperkalaemia, hypocalcaemia and acidosis. Blood products should be immediately available for resuscitation and consideration given to aggressive pre-emptive resuscitation with blood products, calcium bicarbonate and sodium bicarbonate.

Once REBOA is no longer required, the sheath should be flushed and removed once coagulation is normalised and according to local institutional procedure. Proactive thrombectomy or angiography for thrombus associated with the balloon catheter and femoral arterial sheath should be performed in all patients. Its presence is very likely in our experience and complications common if not appropriately managed [12]. Whether the femoral arterial sheath is removed or remains in-situ, careful regular limb vascular observations should be continued as there is a high likelihood of thrombus and delayed ischaemic lower-limb complications.

\section{Scene Management and Human Factors}

It is expected that the operator performing REBOA will become 'task focused'. A clear dialogue is essential between members of the pre-hospital team confirming the REBOA operator will be 'eyes down' on the procedure, leaving the remaining members to take charge of the scene. This is essential to ensure the many other vital and time critical interventions are being concurrently performed and the team is working efficiently. The procedure packs are organised in such a way that an untrained assistant who is unfamiliar with the technique should be able to provide useful assistance. We have learnt, however, that the procedure is quicker and more likely to succeed when performed by a trained operator and a trained assistant. We aim to complete the procedure with needle in skin to balloon inflation within 4-6 minutes, although this is case dependent. The clinician running the scene should also be wary of procedure and scene time and communicate appropriately if delays occur.

\section{GOVERNANCE AND TRAINING}

This is a procedure which is performed on rare occasions in critically injured patients and therefore requires considered application. LAA team members are required to go through rigorous assessment before they are permitted to perform REBOA and then undertake regular simulation-based training. The Institute of Pre-hospital Care has developed the Pre-hospital and Emergency Endovascular Resuscitation (PEER) course which consolidates a formal two-day education programme in endovascular resuscitation theory with practical training. This is then cemented by a period of time working in the service and an assessment of competency including knowledge of our SOP, relevant literature and anatomy and a 'real-time' high-pressure practical scenario.

The introduction and development of this technique was subject to a stringent and multi-level governance process involving all members of the multi-disciplinary working group. This ensures that indications and response to the procedure are reviewed regularly, any complications detected early and that any lessons learned are acted on, continually evolving the process.

\section{LESSONS LEARNED USING THIS INITIAL TECHNIQUE}

LAA was the first known trauma service to use REBOA in the pre-hospital phase. At the time this strategy was being developed, the equipment available for REBOA was limited, with a relative lack of bespoke devices. The technique described here was developed using expertise in endovascular practice drawn from other applications. However, this equipment is relatively cheap and readily available.

Securing rapid, safe vascular access is a primary and essential step in any endovascular procedure. This has been shown to be a practically challenging and rate limiting step in performing REBOA [29] with an associated morbidity [30]. Performing USS guided percutaneous vascular access in hypovolaemic patients in the prehospital phase presents a unique set of challenges. These motor skills can be improved with practice [31] and we aim to increase proficiency with this skill formally through the PEER course and with a focus on regular training. One great advantage of this technique is that it can be performed using a 7Fr sheath which has lower puncture site morbidity compared with devices requiring a larger access sheath [32].

Not using a bespoke device for REBOA results in this initial method being a multistep process. These steps all add to the time taken to perform the procedure. It also makes learning the process more difficult and requires familiarity with more steps and more individual pieces of equipment. Transporting, preparing and using each piece in the correct order all becomes more difficult and adds to the cognitive burden of the team in an already high-stress environment. Using stiff guidewires can present their own benefits and challenges. They carry a risk of an iatrogenic vessel injury or dissection, as well as potential misplacement with blind passing. However, using a wire does offer secure access once established, versatility in exchange of endovascular device and may offer technical familiarity to operators.

A practical point we experienced following balloon inflation is often there is a lack or even absence of balloon migration towards the aortic bifurcation if systolic pressure is low. This carries the risk of placing the balloon too proximally, or the desire to pull the balloon back, which can result in misplacement in an iliac vessel or potentially a vascular injury. This can be mitigated by 
Table 1 Features of exsanguinating heamorrhage - 'the hateful eight' [24].

\begin{tabular}{|c|c|}
\hline Case Features & Clinical Signs of Exsanguinating Pelvic Haemorrhage \\
\hline \multirow{2}{*}{$\begin{array}{l}\text { 1. Mechanism featuring large energy transfer or penetrating injury } \\
\text { to the pelvis or groin }\end{array}$} & 1. Pallor \\
\hline & 2. Clammy \\
\hline \multirow{2}{*}{$\begin{array}{l}\text { 2. Injury pattern compatible with vascular injury and major } \\
\text { haemorrhage }\end{array}$} & 3. Venous collapse \\
\hline & 4. Air hunger \\
\hline \multirow{4}{*}{ 3. Rapid evolution of physiological shock state } & 5. Hypotension (low volume/absent peripheral pulses) \\
\hline & 6. Low/falling End Tidal $\mathrm{CO}_{2}$ \\
\hline & 7. Tachy-or bradycardia \\
\hline & 8. Altered mental state. \\
\hline
\end{tabular}

Table 2 Equipment required for Zone III Pre-hospital REBOA. Divided into four separate, labelled packs to facilitate performance and assistance on scene.

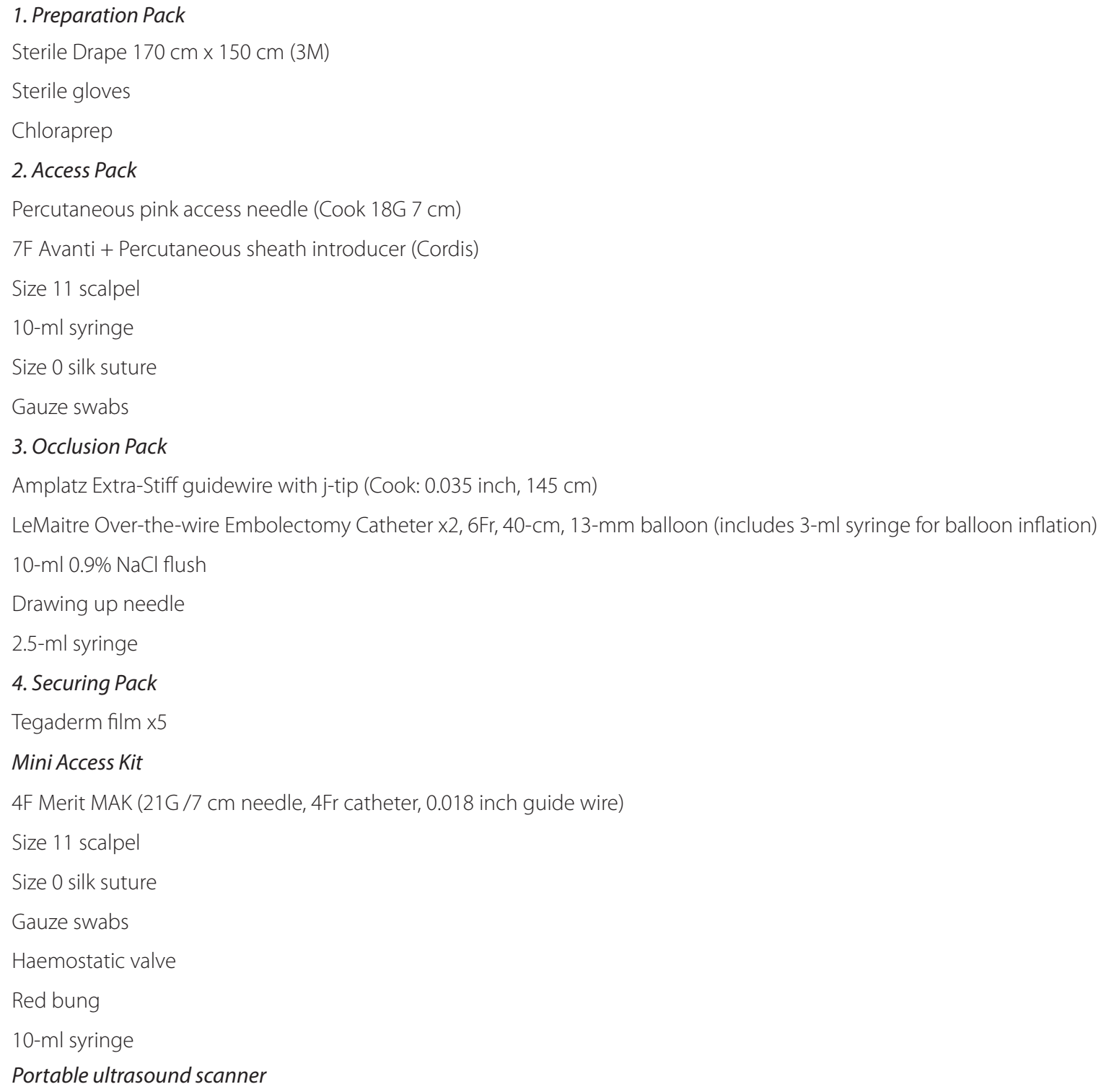


using external landmarks and morphometry to estimate desired insertion distance.

In our experience, there appears to be some inherent partial occlusion property to this balloon, which is evidenced by the presence of computed tomography contrast distal to the site of occlusion in a small number of patients imaged in our case series [33]. There is no way to quantify or control this in real terms using the equipment described, but this may offer some degree of safety while familiarity is gained in establishing a service compared with less compliant and fully occlusive devices.

One major limitation with this strategy is that it only allows occlusion in zone III. We recognise that LAA sees patients who could potentially benefit from supracoeliac (zone I) occlusion. It is thought that some cases with haemorrhage amenable to zone III occlusion were missed due to diagnostic uncertainty. Clinicians found it hard, at times, to commit to the anatomical level of haemorrhage and therefore did not deploy REBOA due to diagnostic doubt. In some of these cases it was noted, retrospectively, that a zone III balloon may have prevented exsanguination. This was a recognised and accepted risk due to the safety considerations and equipment limitations mentioned previously. However, we have learned valuable lessons on careful, detailed assessment and considered the value of other diagnostic tools in differentiating the source of haemorrhage in these cases. We have now progressed to a procedure able to target zone I or III but with extreme caution and with the default that, if unsure of the source of haemorrhage, the clinician will begin with zone III occlusion, only progressing to zone I if the response is inadequate. This has followed our embedding of experience in the procedure, careful monitoring of results and as smaller profile devices capable of zone I occlusion have been developed [29]. More proximal occlusion is accompanied by greater risk with distal ischaemia, and the safest way to mitigate against this is an area of ongoing study. One recommended strategy is early partial REBOA [34, 35], which could not be accurately achieved using the described equipment.

In the evolution of pre-hospital REBOA within our service, progression to a more user-friendly device and technique giving the clinician versatility in occlusion zone and strategy has been key in our aim to replace the need for thoracotomy to treat exsanguinating subdiaphragmatic haemorrhage.

\section{CONCLUSION}

Hybrid endovascular techniques are becoming an increasingly accepted part of the management of critically injured patients. Pre-hospital REBOA provides temporary haemorrhage control and permits transfer for definitive care. We have described the first known technique for introducing pre-hospital REBOA, discussed the reasons why this was implemented and commented on the problems encountered with this approach. The technique described uses 'off-the-shelf' equipment which is familiar to many clinicians with endovascular practice. The relative availability and lower cost of this technique offers some versatility and could be applied in the management of bleeding in other areas such as high-risk labour or postpartum haemorrhage [13,36], as well as use in austere healthcare settings [37] and in those with more limited budgets/resources, or in the management of out-of-hospital cardiac arrest [14].

It should be stressed that this article is not intended to simply be a guide to instruct clinicians on how to perform pre-hospital REBOA. The importance of experience in the recognition of true exsanguinating haemorrhage, the overall context being within a robust training programme supporting meticulous technique, and a multi-level governance programme as part of a prepared trauma system is imperative.

LAA has successfully implemented a pre-hospital zone III REBOA service and we describe how this was practically undertaken as well as reflecting on its strengths and limitations. We recognise that, in order to further develop pre-hospital REBOA, novel strategies and devices permitting zone I occlusion and refined occlusion strategies should be adopted and will be the subject of ongoing study and validation.

\section{Ethics Statement}

(1) All the authors mentioned in the manuscript have agreed to authorship, read and approved the manuscript, and given consent for submission and subsequent publication of the manuscript.

(2) The authors declare that they have read and abided by the JEVTM statement of ethical standards including rules of informed consent and ethical committee approval as stated in the article.

\section{Conflicts of Interest}

The authors declare that they have no conflicts of interest.

\section{Funding}

The authors received no financial support for the research, authorship, and/or publication of this article.

\section{REFERENCES}

[1] Kauvar DS, Lefering R, Wade CE. Impact of hemorrhage on trauma outcome: an overview of epidemiology, clinical presentations, and therapeutic considerations. J Trauma Acute Care Surg. 2006;60:S3-11.

[2] Perkins ZB, De'ath HD, Aylwin C, et al. Epidemiology and outcome of vascular trauma at a British major trauma centre. Eur J Vasc Endovasc Surg. 2012;44: 203-9. 
[3] Perkins ZB, Maytham GD, Koers L, et al. Impact on outcome of a targeted performance improvement programme in haemodynamically unstable patients with a pelvic fracture. Bone Joint J. 2014;96-B:1090-7.

[4] Sadek S, Lockey DJ, Lendrum RA, Perkins Z, Price J, Davies GE. Resuscitative endovascular balloon occlusion of the aorta (REBOA) in the pre-hospital setting: an additional resuscitation option for uncontrolled catastrophic haemorrhage. Resuscitation. 2016;107:135-8.

[5] Alarhayem AQ, Myers JG, Dent D, et al. Time is the enemy: mortality in trauma patients with hemorrhage from torso injury occurs long before the "golden hour". Am J Surg. 2016;212:1101-5.

[6] Marsden M, Carden R, Navaratne L, et al. Outcomes following trauma laparotomy for hypotensive trauma patients: A UK military and civilian perspective. J Trauma Acute Care Surg. 2018;85:620-5.

[7] Rehn M, Davies G, Lockey D. A practical approach to resuscitative thoracotomy. Surgery. 2015;33:455-8.

[8] Konig T, Perkins Z, Davies G. Training non-surgeons to perform resuscitative thoracotomy. Scand J Trauma Resusc Emerg Med. 2013;21:A2.

[9] Nevins EJ, Moori PL, Smith-Williams J, Bird NTE, Taylor JV, Misra N. Should pre-hospital resuscitative thoracotomy be reserved only for penetrating chest trauma? Eur J Trauma Emerg Surg. 2018;44:811-8.

[10] Mancini A, Bonne A, Pirvu A, et al. Retrospective study of thoracotomy performed in a French level 1-trauma center. J Visc Surg. 2017;154:401-6.

[11] Hughes M, Perkins Z. Outcomes following resuscitative thoracotomy for abdominal exsanguination, a systematic review. Scand J Trauma Resusc Emerg Med. 2020;28:9.

[12] Lendrum R, Perkins Z, Chana M, et al. Pre-hospital resuscitative endovascular balloon occlusion of the aorta (REBOA) for exsanguinating pelvic haemorrhage. Resuscitation. 2019;135:6-13.

[13] Stensaeth KH, Sovik E, Haig IN, Skomedal E, Jorgensen A. Fluoroscopy-free resuscitative endovascular balloon occlusion of the aorta (REBOA) for controlling life threatening postpartum hemorrhage. PLoS One. 2017;12:e0174520.

[14] Brede JR, Lafrenz T, Klepstad P, et al. Feasibility of pre-hospital resuscitative endovascular balloon occlusion of the aorta in non-traumatic out-of-hospital cardiac arrest. J Am Heart Assoc. 2019;8:e014394.

[15] Morrison JJ, Galgon RE, Jansen JO, et al. A systematic review of the use of resuscitative endovascular balloon occlusion of the aorta in the management of hemorrhagic shock. J Trauma Acute Care Surg. 2016;80: 324-34.

[16] Gupta BK, Khaneja SC, Flores L, et al. The role of intra-aortic balloon occlusion in penetrating abdominal trauma. J Trauma. 1989;29:861-5.

[17] Martinelli T, Thony F, Declety P, et al. Intra-aortic balloon occlusion to salvage patients with life threatening haemorrhagic shocks from pelvic fractures. J Trauma. 2010;68:942-8.

[18] Brenner ML, Moore LJ, DuBose JJ, et al. A clinical series of resuscitative endovascular balloon occlusion of the aorta for haemorrhage control and resuscitation. J Trauma Acute Care Surg. 2013;75:506-11.
[19] Brenner M, Inaba K, Aiolfi A, et al. Resuscitative endovascular balloon occlusion of the aorta and resuscitative thoracotomy in select patients with hemorrhagic shock: early results from the American Association for the Surgery of Trauma Aortic Occlusion in Resuscitation for Trauma and Acute Care Surgery Registry. J Am Coll Surg. 2018;226:730-40.

[20] White JM, Cannon JW, Stannard A, Markov NP, Spencer JR, Rasmussen TE. Endovascular balloon occlusion of the aorta is superior to resuscitative thoracotomy with aortic clamping in a porcine model of hemorrhagic shock. Surgery. 2011;150:400-9.

[21] Morrison JJ, Percival TJ, Markov NP, et al. Aortic balloon occlusion is effective in controlling pelvic hemorrhage. J Surg Res. 2012;177:341-7.

[22] Markov NP, Percival TJ, Morrison JJ, et al. Physiologic tolerance of descending thoracic aortic balloon occlusion in a swine model of hemorrhagic shock. Surgery. 2013;153:848-56.

[23] Scott DJ, Eliason JL, Villamaria C, et al. A novel fluoroscopy-free, resuscitative endovascular aortic balloon occlusion system in a model of hemorrhagic shock. J Trauma Acute Care Surg. 2013;75:122-8.

[24] Park TS, Batchinsky AI, Belenkiy SM, et al. Resuscitative endovascular balloon occlusion of the aorta (REBOA): comparison with immediate transfusion following massive hemorrhage in swine. J Trauma Acute Care Surg. 2015;79:930-6.

[25] DuBose et al. The AAST prospective Aortic Occlusion for Resuscitation in Trauma and Acute Care Surgery (AORTA) registry: data on contemporary utilization and outcomes of aortic occlusion and resuscitative balloon occlusion of the aorta (REBOA). J Trauma Acute Care Surg. 2016;81:409-19.

[26] Stannard A, Eliason JL, Rasmussen TE. Resuscitative endovascular balloon occlusion of the aorta (REBOA) as an adjunct for hemorrhagic shock. J Trauma. 2011; 71:1869-72.

[27] UK REBOA Trial. The UK-REBOA (resuscitative endovascular balloon occlusion of the aorta) trial. https:// w3.abdn.ac.uk/hsru/reboa/public/public/index.cshtml. Accessed May 2021.

[28] Lendrum R, Perkins Z, Chana M, et al. Reply to: prehospital REBOA: time to clearly define the relevant indications. Resuscitation. 2019;142:191-2.

[29] Romagnoli A, Teeter W, Pasley J, et al. Time to aortic occlusion: it's all about access. J Trauma Acute Care Surg. 2017;83:1161-4.

[30] Manzano-Nunez R, Orlas CP, Herrera-Escobar JP, et al. A meta-analysis of the incidence of complications associated with groin access after the use of resuscitative endovascular balloon occlusion of the aorta in trauma patients. J Trauma Acute Care Surg. 2018;85:626-34.

[31] Borger van der Burg BLS, Maayen RCLA, van Dongen TTCF, et al. feasibility study vascular access and REBOA placement: from zero to hero. J Spec Oper Med. 2018; 18:70-4.

[32] Matsumura Y, Matsumoto J, Kondo H, DIRECT-IABO Investigators, et al. Fewer REBOA complications with smaller devices and partial occlusion: evidence from a multicentre registry in Japan. Emerg Med J. 2017; 34:793-9. 
[33] Pleming W, Kyaw Tun J, Chana M, Jaffer OS, Grier G, Fotheringham T. Imaging trauma patients with resuscitative endovascular balloon occlusion of the aorta (REBOA) inserted in the pre-hospital setting. https:// cslide.ctimeetingtech.com/cirse2018/attendee/eposter/ poster/78209. Accessed May 2021.

[34] Russo RM, Neff LP, Lamb CM, et al. Partial resuscitative endovascular balloon occlusion of the aorta in swine model of hemorrhagic shock. J Am Coll Surg. 2016;223:359-68.

[35] Russo RM, White JM, Baer DG. Partial resuscitative endovascular balloon occlusion of the aorta: a systematic review of the preclinical and clinical literature. J Surg Res. 2021;262:101-4.

[36] Manzano-Nunez R, Escobar-Vidarte MF, Naranjo MP, et al. Expanding the field of acute care surgery: a systematic review of the use of resuscitative endovascular balloon occlusion of the aorta (REBOA) in cases of morbidly adherent placenta. Eur J Trauma Emerg Surg. 2018;44:519-26.

[37] Stokes SC, Theodorou CM, Zakaluzny SA, DuBose JJ, Russo RM. Resuscitative endovascular balloon occlusion of the aorta in combat casualties: the past, present, and future. J Trauma Acute Care Surg. 2021;91(2S Suppl 2):S56-S64. 